\title{
Generalized Jordan-Wigner Transformations
}

\author{
C.D. Batista and G. Ortiz \\ Theoretical Division, Los Alamos National Laboratory, Los Alamos, NM 87545
}

(Received October 23, 2018)

\begin{abstract}
We introduce a new spin-fermion mapping, for arbitrary spin $S$ generating the $S U(2)$ group algebra, that constitutes a natural generalization of the Jordan-Wigner transformation for $S=\frac{1}{2}$. The mapping, valid for regular lattices in any spatial dimension $d$, serves to unravel hidden symmetries in one representation that are manifest in the other. We illustrate the power of the transformation by finding exact solutions to lattice models previously unsolved by standard techniques. We also present a proof of the existence of the Haldane gap in $S=1$ bilinear nearest-neighbors Heisenberg spin chains and discuss the relevance of the mapping to models of strongly correlated electrons. Moreover, we present a general spin-anyon mapping for the case $d \leq 2$.
\end{abstract}

Introduction. Theories of magnetism in normal matter are direct manifestations of quantum mechanics. The vast majority of phenomena occurring in magnets, such as different types of magnetically ordered states, are often simply described by interacting quantum spins [1]. Although quantum spins do not behave as either pure boson or fermion operators, different representations have invoked such particle statistics, for example, the HolsteinPrimakoff, Schwinger, and Dyson-Maleev boson representations for arbitrary spin $S$, or the Jordan-Wigner and Majorana fermion representations for spin $S=\frac{1}{2}$ magnets.

Sometimes it is extremely convenient to reformulate a difficult strongly correlated problem in a way that it becomes more manageable; in some cases there is an exact dualism. This is the idea behind the bosonization techniques and the different algebra representations of a physical problem. The point is that these different representations help us understand various aspects of the same problem by transforming intricate interaction terms into simpler ones. Often, fundamental symmetries which are hidden in one representation are manifest in the other and, moreover, problems which seem untractable can even be exactly solved after the mapping. The simplest and perhaps most popular example is the equivalence between the Heisenberg-Ising $S=\frac{1}{2}$ XXZ chain and a model of interacting spinless fermions through the Jordan-Wigner transformation [2].

The Jordan-Wigner transformation involves the $S=\frac{1}{2}$ irreducible representation of the Lie group $S U(2)$. Here we generalize this spin-fermion mapping to any irreducible representation of dimension $2 S+1$. The three generators $S_{j}^{\mu}(\mu=x, y, z)$ of the Lie algebra for each lattice site $j$ satisfy the commutation relations [3]

$$
\left[S_{j}^{\mu}, S_{k}^{\nu}\right]=i \delta_{j k} \epsilon_{\mu \nu \lambda} S_{j}^{\lambda}
$$

with $\epsilon$ the totally antisymmetric tensor. The algebra generated by the (linear and Hermitian operators) $S_{j}^{\mu}$ is the enveloping algebra of the group $S U(2)$. Equivalently, in terms of the ladder operators $S_{j}^{ \pm}=S_{j}^{x} \pm i S_{j}^{y}$

$$
\begin{aligned}
{\left[S_{j}^{+}, S_{j}^{-}\right] } & =2 S_{j}^{z},\left[S_{j}^{z}, S_{j}^{ \pm}\right]= \pm S_{j}^{ \pm}, \\
\left\{S_{j}^{+}, S_{j}^{-}\right\} & =2\left(S(S+1)-\left(S_{j}^{z}\right)^{2}\right) .
\end{aligned}
$$

We start by analyzing the one-dimensional $S=1$ case. Then, we will show a generalization to arbitrary spin and spatial dimension $d$.

$S=1$ Mapping. We introduce the following composite operators

$$
f_{j}^{\dagger}=\bar{c}_{j 1}^{\dagger}+\bar{c}_{j \overline{1}}, f_{j}=\bar{c}_{j 1}+\bar{c}_{j \overline{1}}^{\dagger},
$$

written in terms of the Hubbard operators $\bar{c}_{j \sigma}^{\dagger}=c_{j \sigma}^{\dagger}(1-$ $\left.\bar{n}_{j \bar{\sigma}}\right)$ and $\bar{c}_{j \sigma}=\left(1-\bar{n}_{j \bar{\sigma}}\right) c_{j \sigma}(\sigma=1,-1)$, which form a subalgebra of the so-called double graded algebra $\operatorname{Spl}(1,2)$ [A]. [A bar in a subindex means the negative of that number (e.g., $\bar{\sigma}=-\sigma)$ ).] For spins on a lattice we fermionize the spins and reproduce the correct spin algebra with the following transformation

$$
\begin{aligned}
S_{j}^{+} & =\sqrt{2}\left(\bar{c}_{j 1}^{\dagger} K_{j}+K_{j}^{\dagger} \bar{c}_{j \overline{1}}\right), \\
S_{j}^{-} & =\sqrt{2}\left(K_{j}^{\dagger} \bar{c}_{j 1}+\bar{c}_{j \overline{1}}^{\dagger} K_{j}\right), \\
S_{j}^{z} & =\bar{n}_{j 1}-\bar{n}_{j \overline{1}},
\end{aligned}
$$

whose inverse manifests the nonlocal character of the mapping

$$
\begin{aligned}
f_{j}^{\dagger} & =\frac{1}{\sqrt{2}} \exp \left[i \pi \sum_{k<j}\left(S_{k}^{z}\right)^{2}\right] S_{j}^{+}, \\
f_{j} & =\frac{1}{\sqrt{2}} \exp \left[-i \pi \sum_{k<j}\left(S_{k}^{z}\right)^{2}\right] S_{j}^{-}, \\
\bar{c}_{j 1}^{\dagger} & =S_{j}^{z} f_{j}^{\dagger}, \quad \bar{c}_{j 1}=f_{j} S_{j}^{z}, \\
\bar{c}_{j \overline{1}}^{\dagger} & =-S_{j}^{z} f_{j}, \quad \bar{c}_{j \overline{1}}=-f_{j}^{\dagger} S_{j}^{z},
\end{aligned}
$$

where the string operators $K_{j}=\exp \left[i \pi \sum_{k<j} \bar{n}_{k}\right]=$ $\prod_{k<j} \prod_{\sigma}\left(1-2 \bar{n}_{j \sigma}\right)$, and the number operators $\bar{n}_{k}=$ $\bar{n}_{k 1}+\bar{n}_{k \overline{1}}$. These $f$-operators have the remarkable property that

$$
\left\{f_{j}^{\dagger}, f_{j}\right\}=\left\{S_{j}^{+}, S_{j}^{-}\right\}
$$


which suggests an analogy between spin operators and "constrained" fermions.

Half-odd integer spin chains have a qualitatively different excitation spectrum than integer spin chains. The Lieb, Schultz, Mattis and Affleck theorem [5] establishes that the half-odd integer antiferromagnetic (AF) bilinear nearest-neighbors (NN) Heisenberg chain is gapless. The same model with integer spins is conjectured to display a Haldane gap [6]. To understand the origin of the Haldane gap we analyze the form of the $1 d S=1$ XXZ Hamiltonian using the above representation (an overall omitted constant $J>0$ determines the energy scale)

$$
\begin{aligned}
H_{\mathrm{xxz}} & =\sum_{j} S_{j}^{z} S_{j+1}^{z}+\Delta\left(S_{j}^{x} S_{j+1}^{x}+S_{j}^{y} S_{j+1}^{y}\right) \\
& =\sum_{j} H_{j}^{z}+H_{j}^{\mathrm{xx}} .
\end{aligned}
$$

It is easy to show that the constrained fermion version of this Hamiltonian is a $\left(S=\frac{1}{2}\right) t-J_{z}$ model [7] plus a particle non-conserving term which breaks the $U(1)$ symmetry

$$
\begin{aligned}
H_{\mathrm{xxz}} & =\sum_{j}\left(\bar{n}_{j 1}-\bar{n}_{j \overline{1}}\right)\left(\bar{n}_{j+11}-\bar{n}_{j+1 \overline{1}}\right) \\
& +\Delta \sum_{j \sigma}\left(\bar{c}_{j \sigma}^{\dagger} \bar{c}_{j+1 \sigma}+\bar{c}_{j \sigma}^{\dagger} \bar{c}_{j+1 \bar{\sigma}}^{\dagger}+\text { H.c. }\right) .
\end{aligned}
$$

In the isotropic $\Delta=1$ limit, $H_{\mathrm{xxz}}$ can be written in a compact way

$$
H_{\text {Heisenberg }}=\sum_{j}\left(\Psi_{j}^{\dagger} \overrightarrow{\mathbf{S}} \Psi_{j}\right) \cdot\left(\Psi_{j+1}^{\dagger} \overrightarrow{\mathbf{S}} \Psi_{j+1}\right)
$$

where $\overrightarrow{\mathbf{S}}$ is an irreducible matrix representation of $S=1$ $(3 \times 3$ matrices $)$ while $\Psi_{j}^{\dagger}$ is the $(1 \times 3)$ vector

$$
\Psi_{j}^{\dagger}=\left[\bar{n}_{j 1},\left(\bar{c}_{j 1}^{\dagger}+\bar{c}_{j \overline{1}}^{\dagger}\right) K_{j}, \bar{n}_{j \overline{1}}\right]
$$

The charge spectrum of the $\left(S=\frac{1}{2}\right) t-J_{z}$ model is gapless but the spin spectrum is gapped due to the explicitly broken $S U(2)$ symmetry (Luther-Emery liquid) [7]. Therefore, the spectrum of the $S=1$ Hamiltonian associated to the $t-J_{z}$ model (which has only spin excitations) is gapless. Hence the term which explicitly breaks $U(1)$ must be responsible for the opening of the Haldane gap. We can prove this by considering the perturbative effect that the interaction $\eta \sum_{j \sigma}\left(\bar{c}_{j \sigma}^{\dagger} \bar{c}_{j+1 \bar{\sigma}}^{\dagger}+\right.$ H.c. $)$ has on the $t$ - $J_{z}$ Hamiltonian. To linear order in $\eta(>0)$, Eq. 7 maps onto the $\left(S=\frac{1}{2}\right)$ XYZ model with $\mathcal{J}_{x}=2+\eta$, $\mathcal{J}_{y}=2-\eta$, and $\mathcal{J}_{z}=-1$. From exact solution of this model [8], it is seen that the system is critical only when $\eta=0$ while for $\eta \neq 0$ a gap to all excitations opens.

$S=1$ Integrable Models [9]. To illustrate further the power of our spin-fermion mapping we now present exact solutions of $1 d S=1$ models that have not been discovered by traditional techniques. These models correspond to the family of bilinear-biquadratic Hamiltonians

$$
\begin{aligned}
H_{1}(\Delta) & =\sum_{j} H_{j}^{z}+H_{j}^{\mathrm{xx}}+\left\{H_{j}^{z}, H_{j}^{\mathrm{xx}}\right\} \\
& =\sum_{j} H_{j}^{z}+\Delta \sum_{\sigma} \bar{c}_{j \sigma}^{\dagger} \bar{c}_{j+1 \sigma}
\end{aligned}
$$

that can be mapped onto a $\left(S=\frac{1}{2}\right) t-J_{z}$ Hamiltonian, whose quantum phase diagram has recently been exactly solved [7].

Another well-studied class of bilinear-biquadratic $S U(2)$ invariant Hamiltonians is 10

$$
H_{2}(\Delta)=\sum_{j} \mathbf{S}_{j} \cdot \mathbf{S}_{j+1}+\Delta\left(\mathbf{S}_{j} \cdot \mathbf{S}_{j+1}\right)^{2}
$$

for $-1 \leq \Delta \leq 1$. The pure Heisenberg $(\Delta=0)$ and Valence Bond Solid models $\left(\Delta=\frac{1}{3}\right)$ belong to the Haldane gapped phase, which extends over the whole interval except at the boundaries $\Delta= \pm 1$ that are quantum critical points. The case $\Delta=-1$ is known to be Bethe ansatz soluble with a unique ground state and gapless. For $\Delta=1$ we can map $H_{2}(1)$ onto the supersymmetric $\left(S=\frac{1}{2}\right) t-J$ Hamiltonian plus a NN repulsive interaction

$$
\begin{aligned}
H_{2}(1) & =-\sum_{j \sigma}\left(\bar{c}_{j \sigma}^{\dagger} \bar{c}_{j+1 \sigma}+\text { H.c. }\right)+2 \sum_{j} \mathbf{s}_{j} \cdot \mathbf{s}_{j+1} \\
& +2 \sum_{j}\left(1-\bar{n}_{j}+\frac{3}{4} \bar{n}_{j} \bar{n}_{j+1}\right),
\end{aligned}
$$

where $\mathbf{s}_{j}$ represents a $S=\frac{1}{2}$ operator. This model is Bethe-ansatz soluble with a gapless phase [11] and is known as the Lai-Sutherland solution [12].

We now discuss the importance of our generalized Jordan-Wigner transformation in unraveling hidden symmetries of an arbitrary spin Hamiltonian. In Eq. 10, for example, the $S=1 S U(2)$ symmetry is manifest. However, both the $S=\frac{1}{2} S U(2)$ and global $U(1)$ gauge symmetries are hidden. On the other hand, in the transformed Hamiltonian, Eq. 11, these two symmetries are manifested explicitly through rotational invariance and charge conservation. The generators of these symmetries are related through the mapping already introduced. To illustrate this, we consider the $U(1)$ symmetry case. Here the generator of the transformation is $Q=\sum_{j} \bar{n}_{j}$ which maps onto $Q=\sum_{j}\left(S_{j}^{z}\right)^{2}$ in the spin representation. The total group symmetry of the Hamiltonian is $U(3)$.

Generalized Transformation. A general transformation for arbitrary spin and spatial dimension is the following

$\underline{\text { Half-odd integer spin } S}\left(\sigma \in \mathcal{F}_{\frac{1}{2}}=\{-S+1, \ldots, S\}\right)$ : 


$$
\begin{gathered}
S_{j}^{+}=\eta_{\bar{S}} \bar{c}_{j \bar{S}+1}^{\dagger} K_{j}+\sum_{\substack{\sigma \in \mathcal{F}_{\frac{1}{2}} \\
\sigma \neq S^{2}}} \eta_{\sigma} \bar{c}_{j \sigma+1}^{\dagger} \bar{c}_{j \sigma}, \\
S_{j}^{-}=\eta_{\bar{S}} K_{j}^{\dagger} \bar{c}_{j \bar{S}+1}+\sum_{\substack{\sigma \in \mathcal{F}_{\frac{1}{2}} \\
\sigma \neq S^{2}}} \eta_{\sigma} \bar{c}_{j \sigma}^{\dagger} \bar{c}_{j \sigma+1}, \\
S_{j}^{z}=-S+\sum_{\sigma \in \mathcal{F}_{\frac{1}{2}}}(S+\sigma) \bar{n}_{j \sigma}, \\
\bar{c}_{j \sigma}^{\dagger}=K_{j}^{\dagger} L_{\sigma}^{\frac{1}{2}}\left(S_{j}^{+}\right)^{\sigma+S} \mathcal{P}_{j}^{\frac{1}{2}}, \\
\text { where } \quad \mathcal{P}_{j}^{\frac{1}{2}}=\prod_{\tau \in \mathcal{F}_{\frac{1}{2}}} \frac{\tau-S_{j}^{z}}{\tau+S}, L_{\sigma}^{\frac{1}{2}}=\prod_{\tau=-S}^{\sigma-1} \eta_{\tau}^{-1} .
\end{gathered}
$$

$\underline{\text { Integer } \operatorname{spin} S}\left(\sigma \in \mathcal{F}_{1}=\{-S, \ldots,-1,1, \ldots, S\}\right)$ :

$$
\begin{gathered}
S_{j}^{+}=\eta_{0}\left(\bar{c}_{j 1}^{\dagger} K_{j}+K_{j}^{\dagger} \bar{c}_{j \overline{1}}\right)+\sum_{\substack{\sigma \in \mathcal{F}_{1} \\
\sigma \neq-1, S}} \eta_{\sigma} \bar{c}_{j \sigma+1}^{\dagger} \bar{c}_{j \sigma}, \\
S_{j}^{-}=\eta_{0}\left(K_{j}^{\dagger} \bar{c}_{j 1}+\bar{c}_{j \overline{1}}^{\dagger} K_{j}\right)+\sum_{\substack{\sigma \in \mathcal{F}_{1} \\
\sigma \neq-1, S}} \eta_{\sigma} \bar{c}_{j \sigma}^{\dagger} \bar{c}_{j \sigma+1}, \\
S_{j}^{z}=\sum_{\sigma \in \mathcal{F}_{1}} \sigma \bar{n}_{j \sigma}, \\
\bar{c}_{j \sigma}^{\dagger}=K_{j}^{\dagger} L_{\sigma}^{1} \begin{cases}\left(S_{j}^{+}\right)^{\sigma} \mathcal{P}_{j}^{1} & \text { if } \sigma>0, \\
\left(S_{j}^{-}\right)^{\sigma} \mathcal{P}_{j}^{1} & \text { if } \sigma<0,\end{cases} \\
\text { where } \quad \mathcal{P}_{j}^{1}=\prod_{\tau \in \mathcal{F}_{1}} \frac{\tau-S_{j}^{z}}{\tau}, L_{\sigma}^{1}=\prod_{\tau=0}^{|\sigma|-1} \eta_{\tau}^{-1},
\end{gathered}
$$

and $\eta_{\sigma}=\sqrt{(S-\sigma)(S+\sigma+1)}$ (see Fig. 1).

The total number of flavors is $N_{f}=2 S$, and the $S=\frac{1}{2}$ case simply reduces to the traditional Jordan-Wigner transformation. These mappings enforce the condition on the Casimir operator $\mathbf{S}_{j}^{2}=S(S+1)$. The generalized constrained fields

$$
\bar{c}_{j \sigma}^{\dagger}=c_{j \sigma}^{\dagger} \prod_{\tau \in \mathcal{F}_{\alpha}}\left(1-n_{j \tau}\right), \bar{c}_{j \sigma}=\prod_{\tau \in \mathcal{F}_{\alpha}}\left(1-n_{j \tau}\right) c_{j \sigma}
$$

form a subalgebra of the generalized Hubbard double graded algebra, where the "unconstrained" operators $c_{j \sigma}^{\dagger}, c_{j \sigma}$ satisfy the standard fermion anticommutation relations $\left(\alpha=\frac{1}{2}, 1\right.$ depending upon the spin character of the representation). These generalized constrained operators (only single occupancy is allowed) anticommute for different sites

$$
\begin{aligned}
& \left\{\bar{c}_{j \sigma}, \bar{c}_{k \sigma^{\prime}}\right\}=\left\{\bar{c}_{j \sigma}^{\dagger}, \bar{c}_{k \sigma^{\prime}}^{\dagger}\right\}=0, \\
& \left\{\bar{c}_{j \sigma}, \bar{c}_{k \sigma^{\prime}}^{\dagger}\right\}=\delta_{j k} \begin{cases}\prod_{\tau \in \mathcal{F}_{\alpha}}\left(1-\bar{n}_{j \tau}\right) & \text { if } \sigma=\sigma^{\prime}, \\
\tau \neq \sigma & \text { if } \sigma \neq \sigma^{\prime},\end{cases}
\end{aligned}
$$

and their number operators satisfy $\bar{n}_{j \sigma} \bar{n}_{j \sigma^{\prime}}=\delta_{\sigma \sigma^{\prime}} \bar{n}_{j \sigma}$.
The string operators $K_{j}$ introduce nonlinear and nonlocal interactions between the constrained fermions. For $1 d$ lattices $\left(K_{j}=K_{j}^{\dagger},\left[K_{i}, K_{j}\right]=0\right)$ they are the so-called kink operators $K_{j}=\exp \left[i \pi \sum_{k<j} \bar{n}_{k}\right]$, while for $2 d$ [13

$$
\begin{aligned}
& K_{\mathbf{j}}=\exp \left[i \sum_{\mathbf{k}} a(\mathbf{k}, \mathbf{j}) \bar{n}_{\mathbf{k}}\right], \text { with } \\
& \bar{n}_{\mathbf{k}}=\sum_{\sigma \in \mathcal{F}_{\alpha}} \bar{n}_{\mathbf{k} \sigma}=1-\mathcal{P}_{\mathbf{k}}^{\alpha} .
\end{aligned}
$$

Here, $a(\mathbf{k}, \mathbf{j})$ is the angle between the spatial vector $\mathbf{k}-\mathbf{j}$ and a fixed direction on the lattice, and $a(\mathbf{j}, \mathbf{j})$ is defined to be zero. We comment that the $1 d$ kink operators constitute a particular case of Eq. 14 with $a(k, j)=\pi$ when $k<j$ and equals zero otherwise. For $d>2$, the string operators generalize [9] along the lines introduced in Ref. [14.

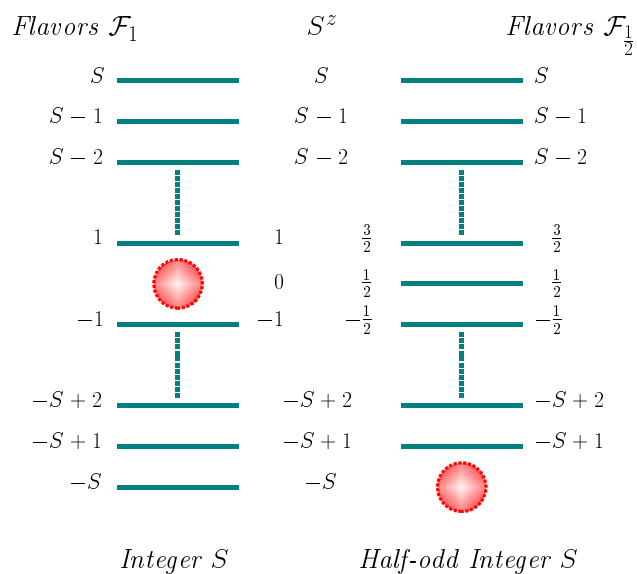

FIG. 1. Constrained fermion states per site for integer and half-odd integer spin $S$. In both cases there are $2 S$ flavors and the corresponding $2 S+1$ values of $S^{z}$ are shown in the middle column. One degree of freedom is assigned to the fermion vacuum (circle) whose relative position depends upon the spin character.

There is always the freedom to perform rotations in spin space to get equivalent representations to the one presented above. However, for bilinear isotropic NN Heisenberg (spin $S U(2)$ rotationally invariant) Hamiltonians in the large- $S$ limit there is a fundamental difference between effective integer and half-odd integer spin cases. In the latter case a new local $U(1)$ gauge symmetry emerges that is explicitly broken in the integer case. For $1 d$ lattices, this is precisely what distinguishes Haldane gap systems [6] from half-odd integer spin chains that are critical.

We mention that other fermionic representations are feasible. In particular, for half-odd integer cases where $2 S+1=\sum_{i=0}^{\bar{N}_{f}}\left(\begin{array}{c}\bar{N}_{f} \\ i\end{array}\right)=2^{\bar{N}_{f}}$ (e.g., $S=\frac{3}{2}$ with $\left.\bar{N}_{f}=2\right)$ a simple transformation in terms of standard "unconstrained" fermions is possible [9]. 
$2 d$ Lattices and Spin-Anyon Mapping. The generalization of these transformations to higher dimensions gives new exact mappings between spin theories and constrained fermion systems in the presence of gauge fields. To illustrate this we write the $S=1$ Hamiltonian $H_{2}(1)$ in the fermion representation for $d=2$

$$
\begin{aligned}
H_{2}(1) & =-\sum_{\mathbf{j} \sigma, \nu}\left(\bar{c}_{\mathbf{j}+\mathbf{e}_{\nu} \sigma}^{\dagger} e^{i A_{\nu}(\mathbf{j})} \bar{c}_{\mathbf{j} \sigma}+\text { H.c. }\right)+2 \sum_{\mathbf{j}, \nu} \mathbf{s}_{\mathbf{j}} \cdot \mathbf{s}_{\mathbf{j}+\mathbf{e}_{\nu}} \\
& +2 \sum_{\mathbf{j}}\left(1-\bar{n}_{\mathbf{j}}+\frac{3}{4} \bar{n}_{\mathbf{j}} \bar{n}_{\left.\mathbf{j}+\mathbf{e}_{\nu}\right)}\right.
\end{aligned}
$$

and

$$
A_{\nu}(\mathbf{j})=\sum_{\mathbf{k}}\left[a(\mathbf{k}, \mathbf{j})-a\left(\mathbf{k}, \mathbf{j}+\mathbf{e}_{\nu}\right)\right] \bar{n}_{\mathbf{k}},
$$

where $\mathbf{e}_{\nu}(\nu=1,2)$ are basis vectors of the Bravais lattice connecting NN and j's represent sites of the corresponding $2 d$ lattice. We note that the field $A_{\nu}(\mathbf{j})$ is associated with the change in particle statistics. It is well-known [13, 1] that the same transmutation of particle statistics can be achieved via a path-integral formulation for $H_{2}(1)$ where an Abelian lattice Chern-Simons term is included. In this formulation a constraint (Gauss's law) requiring that the gauge flux through a plaquette $\mathbf{j}$ be proportional to the total fermion density on the site, $\bar{n}_{\mathbf{j}}$, is enforced. This suggests that our spin-fermion mapping can be generalized to an spin-anyon transformation with a hardcore condition for the anyon fields [9]. In fact, one can formally take our generalized Jordan-Wigner transformation and replace the string operators $K_{\mathbf{j}}$ by the statistical operators $K_{\mathbf{j}}(\theta)=\exp \left[i \theta \sum_{\mathbf{k}} a(\mathbf{k}, \mathbf{j}) \bar{n}_{\mathbf{k}}\right]$ with $0 \leq \theta \leq 1$. With this choice, the $\bar{c}$ operators satisfy equal-time anyon commutation relations $[\theta=1(0)$ corresponds to constrained fermions(bosons)] [9]. Similar ideas apply for $1 d$ lattices.

One immediately sees the relevance of these transformations for the theories of magnetism and hightemperature superconductivity: A class of $S=1$ Hamiltonians that can be mapped onto a lattice-gauge (ChernSimons) $S=\frac{1}{2} t-J$ theory and vice versa, for example, a $S=\frac{1}{2} t-J$ model,

$$
\begin{aligned}
H_{t-J} & =-t \sum_{\mathbf{j} \sigma, \nu}\left(\bar{c}_{\mathbf{j} \sigma}^{\dagger} \bar{c}_{\mathbf{j}+\mathbf{e}_{\nu} \sigma}+\text { H.c. }\right)+J \sum_{\mathbf{j}, \nu} \mathbf{s}_{\mathbf{j}} \cdot \mathbf{s}_{\mathbf{j}+\mathbf{e}_{\nu}} \\
& -\mu \sum_{\mathbf{j}} \bar{n}_{\mathbf{j}}
\end{aligned}
$$

can be exactly mapped onto a lattice-gauge bilinearbiquadratic $S=1$ theory

$$
\begin{aligned}
& H_{t-J}=-\mu \sum_{\mathbf{j}}\left(S_{\mathbf{j}}^{z}\right)^{2}+\frac{J}{8} \sum_{\mathbf{j}, \nu}\left[H_{\mathbf{j} \nu}^{z}-\frac{4 t}{J} S_{\mathbf{j}}^{+} e^{i A_{\nu}(\mathbf{j})} S_{\mathbf{j}+\mathbf{e}_{\nu}}^{-}\right. \\
& \left.-\frac{4 t}{J}\left\{H_{\mathbf{j} \nu}^{z}, S_{\mathbf{j}}^{+} e^{i A_{\nu}(\mathbf{j})} S_{\mathbf{j}+\mathbf{e}_{\nu}}^{-}\right\}+\left(S_{\mathbf{j}}^{+} S_{\mathbf{j}+\mathbf{e}_{\nu}}^{-}\right)^{2}+\text { H.c. }\right] .
\end{aligned}
$$

By means of a semiclassical approximation it has been shown 15 that the ground state of $H_{2}(1)$ is on the boundary between $\operatorname{AF}(\Delta<1)$ and orthogonal nematic (non-uniform, $\Delta>1$ ) phases 15, 10]. These two states are the result of the competition between the quadratic and quartic spin-exchange interactions. In terms of the equivalent $t-J$ gauge theory this translates into a competition between antiferromagnetism and delocalization. Qualitatively, the string-path of the particle moving in an AF background gives rise to a linear confining potential since the number of frustrated magnetic links is proportional to the length of the path. This observation suggests that the inhomogeneous phases observed in the "striped" high- $\mathrm{T}_{c}$ compounds can be driven by the competition between magnetism and delocalization.

Summary. We introduced a general spin-fermion mapping for arbitrary spin $S$ and spatial dimension that naturally generalizes the Jordan-Wigner transformation for $S=\frac{1}{2}$. Mathematically, we established a one-to-one mapping of elements of a Lie algebra onto elements of a fermionic algebra with a hard-core constraint. Several generalizations, like a spin-anyon mapping, and important consequences result from these transformations [9]. Incidentally, we note that there are extremely powerful numerical techniques (cluster algorithms [16]) to study quantum spin systems, and our mapping allows one to extend these methods to study the equivalent fermionic problems.

We thank J.E. Gubernatis for a careful reading of the manuscript. This work was sponsored by the US DOE under contract W-7405-ENG-36.

[1] D.C. Mattis, The Theory of Magnetism I (Springer, Berlin, 1988).

[2] P. Jordan and E. Wigner, Z. Phys. 47, 631 (1928).

[3] For a $d$-dimensional lattice with $N$ sites, the operator $S_{j}^{\mu}$ is defined in terms of a Kronecker product $\otimes$ as

$$
S_{j}^{\mu}=\mathbb{1} \otimes \mathbb{1} \otimes \cdots \otimes \underbrace{S^{\mu}}_{j^{t h}} \otimes \cdots \otimes \mathbb{1},
$$

where $\mathbb{1}$ is the $(2 S+1) \times(2 S+1)$ unit matrix and $S^{\mu}$ is a spin- $S$ operator. Thus $S_{j}^{\mu}$ admits a matrix representation of dimension $(2 S+1)^{N} \times(2 S+1)^{N}$.

[4] A.M. Tsvelik, Quantum Field Theory in Condensed Matter Physics (Cambridge, Cambridge, 1995).

[5] E.H. Lieb, T.D. Schultz, and D.C. Mattis, Ann. Phys. 16, 407 (1961); I. Affleck and E.H. Lieb, Lett. Math. Phys. 12, 57 (1986).

[6] F.D.M. Haldane, Phys. Lett. A 93, 464 (1983).

[7] C.D. Batista and G. Ortiz, cond-mat/0003158.

[8] R.J. Baxter, Exactly Solved Models in Statistical Mechanics (Academic Press, London, 1990). 
[9] More examples and details will be published elsewhere.

[10] G. Fáth and J. Sólyom, Phys. Rev. B 51, 3620 (1995).

[11] P. Schlottmann, Phys. Rev. B 36, 5177 (1987).

[12] C.K. Lai, J. Math. Phys. 15, 1675 (1974); B. Sutherland, Phys. Rev. B 12, 3795 (1975).

[13] E. Fradkin, Phys. Rev. Lett. 63, 322 (1989); D. Eliezer and G.W. Semenoff, Phys. Lett. B 286, 118 (1992).
[14] L. Huerta and J. Zanelli, Phys. Rev. Lett. 71, 3622 (1993).

[15] N. Papanicolaou, Nucl. Phys. B 305, 367 (1988).

[16] J.E. Gubernatis and N. Kawashima, in Monte Carlo and Molecular Dynamics of Condensed Matter Systems (Società Italiana de Fisica, Bologna, 1995) edited by K. Binder and G. Ciccotti. 